\section{DEEP VENOUS THROMBOSIS AND PULMONARY EMBOLISM AFTER LUNG TRANSPLANTATION}

The incidence of deep venous thrombosis or pulmonary embolism after lung or heart-lung transplantation has not been well defined. Pulmonary embolism may be of particular concern in the postoperative period owing to an inadequately developed or absent collateral bronchial circulation and potential risk of pulmonary infarction. Fourteen $(12.1 \%)$ of 116 patients undergoing either lung $(n=87)$ or heart-lung $(n=29)$ transplantation developed thromboembolic complications 10 days to 36 months after operation. Deep vein thrombosis developed in nine patients, including three with upper body thrombosis related to indwelling central venous catheters. Seven patients $(6 \%)$ had pulmonary embolism, and three of them died. Resolution of pulmonary embolism was successfully accomplished by selective pulmonary artery infusion of urokinase in three patients without complications. Our experience indicates that deep vein thrombosis and pulmonary embolism are significant problems after lung transplantation. Mortality is high in those patients in whom pulmonary embolism develops. Therefore, a comprehensive prevention protocol is warranted. (J THORAC CARdiovasc SURg 1995;110:540-4)

Timothy J. Kroshus, MD, Vibhu R. Kshettry, MD, Marshall I. Hertz, MD, and R. Morton Bolman III, MD, Minneapolis, Minn.
V enous thromboembolism is a common occurrence in patients undergoing surgery. It has been estimated that deep venous thrombosis (DVT) and pulmonary embolism (PE) result in 300,000 to 600,000 hospitalizations per year in the United States and that 50,000 to 100,000 of these patients die of PE. 1,2,3 Limited reports are available regarding the incidence of thromboembolism after cardiac surgery or thoracotomy. The incidence of DVT after major surgical operations in patients older than 40 years of age is at least $30 \%$ in the absence of prophylactic measures. ${ }^{4}$ The overall incidence of PE after cardiac operations was noted to be $0.56 \%$ in one report. ${ }^{5}$ Thromboembolic events (DVT/PE) were noted in $26 \%$ of patients after pulmonary resection in another report. ${ }^{3}$ No reports have described the occurrence of thromboembolism after

From the Division of Cardiovascular and Thoracic Surgery, University of Minnesota Hospital and Clinic, Minneapolis, Minn.

Received for publication Sept. 2, 1994.

Accepted for publication Dec. 28, 1994.

Address for reprints: Vibhu R. Kshettry, MD, Division of Cardiovascular and Thoracic Surgery, University of Minnesota, Box 207 UMHC, 420 Delaware St. SE, Minneapolis, MN 55455.

Copyright (C 1995 by Mosby-Year Book, Inc.

$0022-5223 / 95 \$ 3.00+0 \quad \mathbf{1 2 / 1 / 6 3 1 8 0}$ lung transplantation. The purpose of this report is to describe the incidence and course of thromboembolic events in a population of patients undergoing lung transplantation.

\section{Methods}

Patients. The clinical courses of 116 consecutive patients who underwent lung $(n=87)$ or heart-lung $(n=29)$ transplantation at the University of Minnesota Hospital and Clinic between June 1986 and December 1993 were retrospectively reviewed to determine the incidence, diagnostic methods used, treatment, and outcome in those having thromboembolic complications. Fourteen patients had thromboembolic complications, including 11 women and 3 men, with an age range of 5 to 59 years. Patients were categorized by primary disease, transplantation procedure performed, and thromboembolic event (Table I). Pretransplantation risk factors were evaluated, including previous venous thromboembolic events, polycythemia, heart failure, oral contraceptives, and recent myocardial infarction.

Diagnostic methods. All lung transplant recipients underwent postoperative xenon ventilation-perfusion $(\mathrm{V} / \mathrm{Q})$ scanning between postoperative days 7 and 10 as part of our routine posttransplantation evaluation. In addition, follow-up V/Q scans were done at 6 months and 1 year after transplantation. Suspicion of $P E$ prompted a chest radiograph and repeat $V / Q$ scan. $V / Q$ scans were rated as low, indeterminate, or high probability based on Blobiello criteria. Change from a previous $V / Q$ scan was considered significant, as was an unmatched perfusion defect. The diagnosis of $\mathrm{PE}$ was confirmed by pulmonary angiography in four patients and by autopsy in another. Two other 
Table I. Primary disease and complication

\begin{tabular}{llccc}
\hline Procedure & \multicolumn{1}{c}{ Primary disease } & Age & Sex & Complication \\
\hline LSL & Primary pulmonary HTN & 51 & $\mathrm{~F}$ & DVT $\times 4 /$ PE \\
LSL & $\alpha_{1}$-antitrypsin & 45 & $\mathrm{~F}$ & DVT \\
BSL & Idiopathic pulmonary fibrosis & 42 & $\mathrm{~F}$ & DVT \\
LSL & $\alpha_{1}$-antitrypsin & 58 & $\mathrm{~F}$ & PE $\times 2 /$ DVT $\times 2$ \\
LSL & Chronic obstructive pulmonary disease & 58 & $\mathrm{~F}$ & PE \\
LSL & $\alpha_{1}$-antitrypsin & 47 & $\mathrm{M}$ & PE \\
BSL & Primary pulmonary HTN & 30 & $\mathrm{~F}$ & PE \\
LSL & Chronic obstructive pulmonary disease & 46 & $\mathrm{~F}$ & DVT \\
RSL & $\alpha_{1}$-antitrypsin & 58 & $\mathrm{M}$ & DVT \\
HL & $\alpha_{1}$-antitrypsin & 54 & $\mathrm{~F}$ & PE \\
RSL & Idiopathic pulmonary fibrosis & 59 & $\mathrm{M}$ & PE \\
HL & $\alpha_{1}$-antitrypsin & 47 & $\mathrm{~F}$ & DVT \\
LSL; BSL & Primary pulmonary HTN & 4 & $\mathrm{~F}$ & DVT \\
BSL & Primary pulmonary HTN & 40 & $\mathrm{~F}$ & DVT
\end{tabular}

$B S L$, Bilateral single lung transplantation; $D V T$, deep venous thrombosis; $F$, female; $H L$, heart-lung transplantation; $H T N$, hypertension; $L S L$, left single lung transplantation; $M$, male; $P E$, pulmonary embolism; $R S L$, right single lung transplantation.

patients had a presumed diagnosis based on the presence of small unmatched perfusion defects on follow-up V/Q scans that were different from previous V/Q scan findings. These two patients were in clinically stable condition and were treated with anticoagulation with close follow-up. One of these patients was considered for pulmonary angiography, but the test was not performed because of renal insufficiency.

In addition, all patients with suspected PE underwent lower extremity duplex ultrasonic scanning in an attempt to find the origin of the PE. Those with negative results of lower extremity duplex ultrasonography underwent upper body ultrasonography if indwelling central venous catheters were present.

Patients who had extremity swelling underwent duplex ultrasonography for diagnosis of DVT. No patient underwent venography for diagnosis. Close follow-up was performed in those patients with diagnosis of a thromboembolic event. Serial chest roentgenograms and V/Q scans were obtained every 1 to 2 weeks until resolution was noted.

\section{Results}

A total of 14 patients had 21 events, including 7 with DVT, 5 with PE, and 2 with both DVT and PE. One patient had five events (one PE and four DVTs) and another patient had 4 events (two PEs and two DVTs). The former patient was considered to be at increased risk before transplantation because of a history of superficial thrombophlebitis. Patients with DVT had unilateral extremity swelling and pain. Five patients had documented lower extremity DVT and were initially treated with anticoagulation. This was poorly tolerated in two patients because of bleeding complications and recurrence of DVT despite therapeutic anticoagulation parameters, eventually necessitating placement of an inferior vena cava filter (Vena Tech LGM vena caval filter system, B. Braun Biotech, Allentown, Pa.). One patient had a history of DVT and filter placement 6 months before transplantation. This patient had a recurrent DVT in the same location in the proximal left superficial femoral vein 2 months after transplantation. An additional patient was considered to be at possible increased risk because of a history of lower extremity superficial thrombophlebitis. Upper body DVT developed in three patients, including one with left subclavian vein thrombosis and two with right internal jugular vein thrombosis. All upper body DVT episodes were associated with indwelling central venous catheters, and all were treated by catheter removal and anticoagulation with heparin and warfarin. Follow-up duplex ultrasound scanning was performed every 1 to 2 weeks until thrombus resolution was ensured.

Clinical features of patients with PE are summarized in Table II. Of the seven patients experiencing PE, three died (43\%). One died suddenly 22 days after transplantation from a saddle embolism that occurred after the patient was discharged from the hospital. Another patient experienced PE 11 days after transplantation and eventually died on the twenty-first postoperative day of infectious complications and multiple organ failure syndrome. In an additional patient PE developed 2 months after transplantation and was successfully treated with urokinase followed by placement of an inferior vena cava filter. A recurrent PE occurred at 12 months after transplantation, and the patient died 4 weeks later of progressive organ failure and sepsis.

Four patients with PE survived (see Table II). Two demonstrated no deterioration of pulmonary 
Table II. Time after transplant, treatment, and outcome by event

\begin{tabular}{|c|c|c|c|}
\hline Event & $\begin{array}{c}\text { Time after } \\
\text { transplantation }\end{array}$ & Treatment & Outcome \\
\hline $\mathrm{PE} / \mathrm{DVT}$ & 38 days & Anticoagulation & Resolved \\
\hline DVT & $12 \mathrm{mo}$ & Anticoagulation & Resolved \\
\hline DVT & $13 \mathrm{mo}$ & Anticoagulation & Resolved \\
\hline Thrombophlebitis & $36 \mathrm{mo}$ & IVC filter & Resolved \\
\hline DVT & 19 days & IVC filter & Resolved \\
\hline DVT & $22 \mathrm{mo}$ & Anticoagulation & Resolved \\
\hline $\mathrm{PE} / \mathrm{DVT}$ & $2 \mathrm{mo}$ & $\begin{array}{l}\text { Urokinase/ } \\
\text { IVC filter }\end{array}$ & Resolved \\
\hline DVT & 4 mo & Anticoagulation & Resolved \\
\hline PE & 12 mo & Anticoagulation & Death \\
\hline PE & 22 days & Anticoagulation & Death \\
\hline PE & 10 days & $\begin{array}{l}\text { Urokinase/ } \\
\text { IVC filter }\end{array}$ & Death \\
\hline $\mathrm{PE}$ & $12 \mathrm{mo}$ & Anticoagulation & Resolved \\
\hline DVT & $2 \mathrm{mo}$ & $\begin{array}{l}\text { Anticoagulation/I } \\
\text { VC filter }\end{array}$ & Resolved \\
\hline DVT & $2 \mathrm{mo}$ & Anticoagulation & Resolved \\
\hline PE & $8 \mathrm{mo}$ & Urokinase & Resolved \\
\hline PE & $5 \mathrm{mo}$ & $\begin{array}{l}\text { Anticoagulation/ } \\
\text { IVC filter }\end{array}$ & Resolved \\
\hline DVT & $1 \mathrm{mo}$ & $\begin{array}{l}\text { Remove central } \\
\text { line }\end{array}$ & Resolved \\
\hline DVT & $7 \mathrm{mo}$ & $\begin{array}{l}\text { Remove central } \\
\text { line }\end{array}$ & Resolved \\
\hline DVT & 21 days & $\begin{array}{l}\text { Remove central } \\
\text { line }\end{array}$ & Resolved \\
\hline
\end{tabular}

$\overline{D V T \text {, Deep venous thrombosis; } I V C \text {, inferior vena cava; } P E \text {, pulmonary }}$ embolism.

function after recovery from their event, and one later died of bronchiolitis obliterans. The fourth patient died 1 year after the PE event of an unrelated cause. The PE originated from the lower extremity in one patient, from the left upper extremity in one patient, and from thrombus surrounding a central venous catheter in one patient. Origin was unknown in four patients. Presenting symptoms in patients with PE included pleuritic chest pain in two patients and cardiac arrhythmias in two patients (atrial fibrillation in one and supraventricular tachycardia in one); one patient had massive hemoptysis and acute respiratory arrest with immediate death from a saddle embolism.

Treatment of patients after a diagnosis of thromboembolic complications consisted of initial heparinization followed by oral anticoagulation with warfarin, maintaining the prothrombin time at 1.5 to 2 times normal. Three patients underwent 24 to 48 hours of selective urokinase infusion by pulmonary artery catheter placement after confirmation of $\mathrm{PE}$ by pulmonary angiography. There were no docu- mented complications related to the use of urokinase or pulmonary artery catheter placement in any of these patients. Follow-up pulmonary angiography at 12- to 24-hour intervals after initiation of urokinase revealed almost complete resolution of thrombus in two patients and complete resolution in the other patient. These patients were then anticoagulated with heparin followed by warfarin. Those patients not able to tolerate oral anticoagulation or who had additional thromboembolic events while undergoing therapeutic anticoagulation underwent placement of an inferior vena cava filter $(n=4)$. No patient in this review underwent pulmonary embolectomy.

\section{Discussion}

Our findings suggest that lung transplant recipients constitute a high-risk group for DVT and subsequent $\mathrm{PE}$. The significance of this problem is obvious because this group of patients cannot afford the threat of infarction of lung tissue after transplantation. The risk of infarction from a $\mathrm{PE}$ is greatest in the immediate perioperative period because the newly transplanted lung does not have an alternate blood supply owing to the lack of a bronchial circulation. In addition, despite the redevelopment of bronchial collaterals with time, this blood supply may not be sufficient to meet the requirements of the transplanted lung and prevent infarction if a PE occurs later in the postoperative period.

Little information is available regarding the incidence and significance of DVT and PE after lung transplantation. It is known that the incidence of DVT after major surgical operations is at least $30 \%$ for patients older than 40 years of age if no prophylactic measures are used. ${ }^{4}$ This incidence is dramatically reduced with the use of prophylactic measures. The incidence of clinically significant PE has been reported as $1.6 \%$ in the general surgical population, with the likelihood of a major PE leading to death approaching $1 \% .^{6}$ One study that analyzed 144 patients who experienced PE revealed mortality in 12 patients $(8 \%)$; in 4 patients $(3 \%)$, the $\mathrm{PE}$ was determined to be the primary cause of death. ${ }^{7}$

Several acquired and inherited risk factors for the development of DVT and PE have been recognized. Lung transplant recipients often fulfill some of the elements of Virchow's classic triad of stasis, endothelial injury, and hypercoagulability. A period of immobilization and ventilator dependence immediately after transplantation leads to lower extremity venous stasis. In addition, these patients usually have indwelling central venous lines for monitoring 
and access, all of which can predispose patients to DVT. Acquired risk factors predisposing to DVT/PE include major surgical procedures resulting in patient immobilization and medical diseases associated with abnormalities of coagulation and fibrinolysis, platelet abnormalities, and blood vessel disorders. ${ }^{6,8}$ Inherited disorders include protein $\mathrm{C}$ deficiency, ${ }^{9}$ protein S deficiency, ${ }^{10}$ antithrombin III deficiency, ${ }^{11,12}$ dysfibrinogenemia, ${ }^{13}$ and disorders of plasminogen and plasminogen activation. ${ }^{14,15}$ In addition, patients undergoing lung transplantation may have pulmonary hypertension, which may constitute a risk factor, although right-sided heart pressures usually return to near normal levels after transplantation. It is important to recognize those patients at increased risk before transplantation to administer adequate prophylaxis during the perioperative and postoperative periods. Those patients who have had previous thromboembolic events constitute an extremely high-risk group for recurrence. One patient in our series was identified to be at increased risk before transplantation because of a history of DVT, and another was considered to be at increased risk because of a history of superficial thrombophlebitis.

In our series, symptomatic PE developed in 7 $(6 \%)$ of 116 patients, and $3(43 \%)$ of the 7 died. The primary cause of death was determined to be PE in only one patient; the other two patients died 10 days and 4 weeks later, respectively, of infectious complications. Clearly, the PE was the inciting event in the fatal course experienced by each of these patients.

Diagnosis of PE continues to be a difficult problem. A high index of suspicion and low threshold for obtaining diagnostic studies should exist on the basis of clinical impression. Initial investigations consist of a chest roentgenogram, V/Q scan, and lower extremity duplex ultrasonic scanning. If clinical suspicion is high and the V/Q scan shows high probability, patients should undergo pulmonary angiography for definitive diagnosis. Patients who are not candidates for pulmonary angiography should be aggressively treated on the basis of clinical impression and initial diagnostic study results. The difficulty in diagnosing $P E$ remains with those patients who have indeterminate $\mathrm{V} / \mathrm{Q}$ scan results. These patients should be referred for pulmonary angiography if clinical suspicion is high and there is supporting evidence of DVT on duplex ultrasonic scanning. If V/Q scan results are of low probability and duplex ultrasonic scanning results are negative, patients may safely be observed if clinical suspicion is low.
The importance of noninvasive duplex ultrasonography for detection of DVT cannot be overlooked. It has been reported that more than $90 \%$ of PEs originate in the proximal lower extremities. ${ }^{16}$ In our series, a significant number of patients had negative lower extremity duplex ultrasound results but were found to have thrombus surrounding upper body indwelling central venous catheters. We conduct an aggressive search for DVT in both the lower extremities and surrounding central lines in all patients with suspicion of PE by means of duplex ultrasonography. No patient in our series underwent contrast venography for diagnosis of DVT.

If $\mathrm{PE}$ is confirmed angiographically and the thrombus is occlusive of several pulmonary segments, directed pulmonary artery urokinase infusion can be performed successfully, as demonstrated in three of our patients. Urokinase infusion has been shown to be effective when it is administered by a peripheral intravenous route or intraarterially. However, the collateral bronchial circulation may not be adequately developed, and in the lung transplant recipient it is absent. Therefore, we used a directed pulmonary artery infusion in an attempt to obtain clot resolution as quickly as possible because of the potential risk of lung infarction. There was no morbidity or mortality related to pulmonary artery urokinase infusion in these patients.

Prophylaxis of DVT and PE is a critical component of the management of patients after lung transplantation. Subcutaneous heparin and pneumatic compression devices have both been shown to decrease the incidence and subsequent morbidity and mortality of DVT and PE. In general, the use of aspirin has not been shown to add a protective effect, ${ }^{6}$ although the incidence of thromboembolism after thoracotomy for carcinoma was markedly decreased in patients receiving aspirin or ibuprofen in one study. ${ }^{3}$ We have adopted a program of perioperative and postoperative subcutaneous heparin administration extending until the time of discharge. Pneumatic compression garments are used during and after the operation until the patient is ambulatory. After patients are ambulatory, they receive antiplatelet agents. Those patients having thromboembolic events initially receive intravenous heparin until clot resolution, followed by oral anticoagulants (warfarin). Recurrent DVT/PE during therapeutic anticoagulation, intolerance of oral anticoagulants, or presence of a proximal lower extremity DVT in a patient prompts consideration of an inferior vena cava filter.

The true incidence of PE in this patient popula- 
tion is not known. Many patients may have had small and clinically silent PEs, or the PE may lodge in the native lung in patients with single lung transplants in whom clinical symptoms may not be produced. The diagnosis of a PE to the native lung in single lung transplant recipients is an especially difficult problem diagnostically because chest roentgenography and V/Q scan findings are frequently abnormal in the native lung. After diagnosis and treatment of PE or DVT, all patients should undergo close follow-up, with duplex ultrasonography and $\mathrm{V} / \mathrm{Q}$ scanning performed every 1 to 2 weeks until resolution, and then be followed up on a routine basis. Oral anticoagulant medications should be continued indefinitely as long as they are tolerated without serious side effects.

The risk of thromboembolic complications extends well into the postoperative period, including the period after discharge from the hospital. It also appears that the greatest risk for the development of DVT is in the period immediately after prophylaxis is discontinued. In this series, thromboembolic complications occurred from 10 days to 36 months after transplantation, with most events occurring after discharge from the hospital. This delay underscores the importance of continued surveillance and a heightened awareness for these events after the patient has been discharged from hospitalized care. The need for prophylaxis may continue well into the postoperative period.

In summary, thromboembolic complications remain a serious problem for the lung transplant recipient, and a comprehensive prevention protocol is warranted. Prophylaxis needs to extend well into the posttransplantation period, including the period after discharge from the hospital. A high index of suspicion is necessary to allow early diagnosis and successful treatment of these potentially fatal disorders. The true incidence of DVT/PE remains unknown in this patient population and may extend late into the postoperative period, including the period after discharge from the hospital. A multicenter trial to address the efficacy and cost-effectiveness of a follow-up screening program for the detection of clinically silent DVT may be helpful.

\section{REFERENCES}

1. Jones TK, Barnes RW, Greenfield LJ. Greenfield vena caval filter: rationale and current indications. Ann Thorac Surg 1986;42:(Suppl 6):S48.

2. Dalen JE, Alpert JS. Natural history of pulmonary embolism. Prog Cardiovasc Dis 1975;17:259.

3. Ziomek S, Read RC, Tobler G, et al. Thromboembolism in patients undergoing thoracotomy. Ann Thorac Surg 1993;56:223-7.

4. Scurr JH. How long after surgery does the risk of thromboembolism persist? Acta Chir Scand Suppl 1990;556:22-4.

5. Gillinov AM, Davis EA, Alberg AJ, et al. Pulmonary embolism in the cardiac surgical patient. Ann Thorac Surg 1992;53:988-91.

6. Prevention of venous thrombosis and pulmonary embolism (consensus conference). JAMA 1986;256:744-9.

7. Alpert JS, Smith R, Carlson J, et al. Mortality in patients treated for pulmonary embolism. JAMA 1976;236:1477-80.

8. Schafer AI. The hypercoagulable states. Ann Intern Med 1985;102:814-28.

9. Broekmans AW, Veltkamp JJ, Bertina RM. Congenital protein $\mathrm{C}$ deficiency and venous thromboembolism: a study of three Dutch families. N Engl J Med 1983;309:340-4.

10. Comp PC, Esmon CT. Recurrent venous thromboembolism in patients with a partial deficiency of protein S. N Engl J Med 1984;311:1525-8.

11. Winter JH, Fenech A, Ridley W, et al. Familial antithrombin III deficiency. Q J Med 1982;51:373-95.

12. Prochownik EV, Antonarakis S, Bauer KA, et al. Molecular heterogeneity of inherited antithrombin III deficiency. N Engl J Med 1983;308:1549-52.

13. Carrell N, Gabriel DA, Blatt PM, et al. Hereditary dysfibrinogenemia in a patient with thrombotic disease. Blood 1983;62:439-47.

14. Aoki N, Moroi M, Sakata $Y$, et al. Abnormal plasminogen: a hereditary molecular abnormality found in a patient with recurrent thrombosis. J Clin Invest 1978;61:1186-95.

15. Stead NW, Bauer KA, Kinney TR, et al. Venous thrombosis in a family with defective release of vascular plasminogen activator and elevated plasma factor VIII/von Willebrand's factor. Am J Med 1983;74: 33-9.

16. Palevsky HI, Fishman AP. Diagnosis and treatment of pulmonary embolism and deep venous thrombosis. In: Fishman AP, ed. Update: pulmonary diseases and disorders. New York: McGraw-Hill, 1992: $451-64$. 